\title{
Acute infective endocarditis during COVID-19 pandemic time: the dark side of the moon
}

\author{
Roberto Lorusso $^{1}$, Antonio Calafiore ${ }^{2}$, and Michele Di Mauro ${ }^{3}$ \\ ${ }^{1}$ Maastricht University Medical Centre \\ ${ }^{2}$ John Paul II Foundation for Research and Treatment \\ ${ }^{3}$ Maastricht UMC+
}

January 20, 2022

\begin{abstract}
The COVID-19 pandemic has remarkably impacted the hospital management and the profile of patients suffering from acute cardiovascular syndromes. Among them, acute infective endocarditis (AIE) represented a rather frequent part of these urgent/emergent procedures. The paper by $\mathrm{Li}$ and colleagues has clearly shown the higher risk features which patients with diagnosis of AIE presented at hospital admission during the first part (first and second waves) of the outbreak, often requiring challenging operations, but fortunately not associated with worse outcome if compared to results obtained prior to the SARS-2 pandemic. The report discussed herein presents several other aspects worth of discussion and comments, particularly in relation to hospital management and post-discharge outcome which certainly deserve to be highlighted, but also further investigations.
\end{abstract}

\section{Acute infective endocarditis during COVID-19 pandemic time: the dark side of the moon}

Roberto Lorusso $^{1,2}, \mathrm{MD}, \mathrm{PhD}$, Antonio M. Calafiore ${ }^{3}$, MD, and Michele Di Mauro ${ }^{1}, \mathrm{MD}, \mathrm{PhD}, \mathrm{MSc}$

${ }^{1}$ Cardio-Thoracic Surgery Department, Heart and Vascular Centre, Maastricht University Medical Centre, Maastricht, The Netherlands,

${ }^{2}$ Cardiovascular Research Institute Maastricht (CARIM), Maastricht, The Netherlands,

${ }^{3}$ Cardiac Surgery Division, Henry Dunant Hospital, Athens, Greece

Word count: 985

Address for correspondence:

Prof. Dr. Roberto Lorusso, PhD

${ }^{1}$ Cardio-Thoracic Surgery Department, Heart and Vascular Centre,

Maastricht University Medical Centre,

${ }^{2}$ Cardiovascular Research Institute Maastricht (CARIM)

P. Debyelaan 25 - 6202 AZ Maastricht, The Netherlands,

Email:roberto.lorusso@mumc.nl orroberto.lorussobs@gmail.com

Tel. +31433876032

\section{Abstract}


The COVID-19 pandemic has remarkably impacted the hospital management and the profile of patients suffering from acute cardiovascular syndromes. Among them, acute infective endocarditis (AIE) represented a rather frequent part of these urgent/emergent procedures. The paper by $\mathrm{Li}$ and colleagues has clearly shown the higher risk features which patients with diagnosis of AIE presented at hospital admission during the first part (first and second waves) of the outbreak, often requiring challenging operations, but fortunately not associated with worse outcome if compared to results obtained prior to the SARS-2 pandemic. The report discussed herein presents several other aspects worth of discussion and comments, particularly in relation to hospital management and post-discharge outcome which certainly deserve to be highlighted, but also further investigations.

As well-known, besides the unprecedented impact of the COVID-19 worldwide pandemic with regards to patients experiencing a variety of clinical compromise due to the infective illness, lack of dedicated beds and equipment to face overwhelming hospital admittance for respiratory dysfunction, delay in hospital admission of patients with other illnesses, and reduced hospital activities for non-COVID-related treatments, were also described as severe consequences during the outbreak with inevitable suboptimal patient management ${ }^{1-5}$.

$\mathrm{Li}$ and colleagues have reported a single-centre, retrospective, and comparative study looking at two-year series of patients requiring surgical treatment for acute infective endocarditis (AIE) just prior or just after the start of the COVID-19 pandemics ${ }^{6}$. The findings of this study clearly confirm that patients admitted with a suspicion of AIE had a more critical cardiac as well as general clinical conditions at admittance during the pandemic. This translated into higher risk, more complex operations, and longer ICU as well as in-hospital stays. The good news is that this more challenging scenario did not eventually lead to higher in-hospital mortality. This is accordance with the study of Nader and associates who showed a higher risk profile and active endocarditis during the SARS-2 outbreak, characterized also by a more complicated perioperative course $^{4}$, data also confirmed by the Italian national survey ${ }^{3}$.

Despite the reassuring early outcome of the study of $\mathrm{Li}$ and associates, however, there are still some major issues which will deserve further comments and, hopefully, additional investigations and data. Are these patients, despite not worse in-hospital outcome, more prone to recurrence of AIE or other post-discharge complications or even less favorable outcome? Are these patients experiencing additional shortcomings related to the suboptimal pre-operative delayed management and more complex profile? Is the more complex treatment and operations going to influence the post-discharge late clinical conditions? These questions are unfortunately not met in the Li's and associates' investigation and would require ad hoc research.

We are aware that all medical specialties have been somehow hit by the overall COVID-19 illness, particularly during the first pandemic wave, perhaps also potentially affecting the overall quality of care of the treated patients based on the overwhelming pressure exerted by the pandemic itself. Nonetheless, acute cardiovascular syndromes, and, among them, AIEs, may have suffered from a significant cluster of suboptimal organization and management due to the hustles observed in daily health care provision ${ }^{1-3}$.

Another interesting aspect might be related to the in-hospital acquired COVID-19 in these patients, apparently not described by Li and collaborators. Wrong diagnosis of COVID-19 illness, delaying an AIE-related recognition, or the combination of the two conditions, making clinical management even more challenging ${ }^{8}$, have been described. Furthermore, patients treated for AIE were at risk also for in-hospital acquired COVID19 infection, although this event was really limited in the Li's and other series ${ }^{5-7}$.

There is no doubt that all the non-COVID-19 illnesses, particularly the acute cardiovascular syndromes, have been accounted with a sort of shadow or darkness, and, only recently, the SARS-2 COVID "collateral damages" have been coming to the surface, indicating how many of these situations, with patients either suffering from a voluntary or hospital-based delayed admittance or consideration, occurred during the COVID pandemic ${ }^{9}$. Several papers have described the well-known shut-down or remarkable reduction of cardiac surgical programs, with the obvious and sadly known consequences ${ }^{1-3}$. Delayed treatment of acute myocardial infarction, with its potentially fatal or life-threatening complications, or of AIE-related conditions, either for the fear of the patients themselves to refer at the COVID-19 overwhelmed health care structures or initially 
left home to reduce the ongoing patient burden/flow to the "drowning" hospitals, have been described 9

The rather stable number of AIE patients, when comparing the period prior to the pandemic and the first and second waves, as described in the paper of Li and colleagues ${ }^{5}$, should not prompt to the conclusion that COVID-19 was not influencing the overall rate of AIE or nor responsible for such an illness. It is however true that, among the overall cardio-vascular complications attributed to COVID-19 virus, AIE does not represent a widely reported event, rather, no clear relationship has been described or conclusively demonstrated. Interstitial compromise, like capillary microthrombosis, or inflammatory state-related conditions, like acute myocarditis or pericarditis and tamponade, have been the vast majority of conditions reported as far as COVID-19 and cardiac compromised is concerned ${ }^{9,10}$.

Antother important aspect highlighted by the paper of Li and colleagues accounts for the relevance of the multi-disciplinary and dedicated team, namely the Acute Endocarditis Team, plays a critical role in the appropriate management and therapeutical success of such complex patients, as already underlined by other several series/experiences ${ }^{4}$. Today, even amid difficult organizational and hospital-related pressure, technology may help health care professionals to reduce decision-making times and subsequent treatment which may compensate organizational and disease-related uncertainties and delay. Remote or online case-presentation, together with the dedicated team discussion, may favor or partially compensate delayed management, although prompt diagnosis and therapies remain the major contribution to successful acute cardiac diseases.

Obviously, country-based or regional circumstances may constitute additional hustles in the appropriate chain-of-handling of patients with AIE. This has been also properly highlighted by Li and colleagues, showing all the aspects which caused delayed in these difficult times ${ }^{4}$. Likewise, this experience underlines the peculiar circumstances AIE patients may experience, which represent well-known factors predisposing the patients to final potentially lethal conditions, like acute heart failure, septic shock or even cardiac arrest. Finally, the patient transfer organization was certainly also seriously reduced or inefficient based, again, on the dramatically superior number of patients affected by COVID-19, and considered at higher priority with respect to all the other patients. This particular aspect should represent a remarkable and sensitive aspect for the health care administrator and organizations to be able, in the future, to preventively design and arrange crisis-based strategies in order to avoid "privileged" patients, more based on the ongoing emotion rather than actual needs and level of urgency/emergency, as unfortunately observed during the past two years.

\section{References}

1.Fudulu DP, Angelini GD. Cardiac Surgery in the time of the coronavirus. J Card Surg 2020;1-3

2.Harky A, Harrington D, Nawayto O, et al. COVID-19 and cardiac surgery: the perspective from United Kingdom. J Card Surg 2020; . .: :1-10

3.Rubino AS, De Santo LS, Pisano A, Di Mauro M, Benussi S, Borghetti V, Castiglioni A, Chiariello L, Colli A, De Bellis A, et al. Cardiac surgery practice during COVID-19 outbreak: a multicentre national survey. Eur J Cardio-Thorac Surg 2021

4.Nader J, Anselmi A, Tomasi J, Martin A, Aymami M, Rouze S, et al. Adult cardiac surgery during COVID-19 lockdown: impact on activity and outcomes in a high-volume centre. Arch Cardiovasc Dis 2021;114:364-70

5.Shah SMI, Zafar MDB, Yasmin F, Ghazi EM, Jatoi HN, Jawed A, Nadeem A, Khan Z, Anas Z, Siddiqui S. Exploring the impact of the COVID-19 pandemic on cardiac surgical services: a scoping review. J Card Surg 2021

6. Li et al; 2022; epub ahead of print

7.Schizas N, Michailidis T, Samiotis I, Patris V, Papakostantinou K, Argiriou M, Dedeilias P. Delayed diagnosis and treatment of a critically ill patient with infective endocarditis due to a false positive molecular diagnostic test for SARS-CoV-2. Am J Case Rep 2020;21;e925931 
8.Alizadeh K, Bucke D, Khan S. Complex case of COVID-19 and infective endocarditis. Brit Med J 2021;14:e242205

9.Jaffe AS, Cleland JGF, Katus HA. Myocardial injury in severe COVID-19 infection. Eur Heart J 2020;41:2080-2

10.Hanley B, Naresh KN, Roufosse C, et al. Histopathological findings and viral trophism in UK patients with severe fatal COVID-19: a post-mortem study. Lancet Microbe 2020;1:e245-e253 